Journal of Mathematics and Statistics 6 (3): 240-245, 2010

ISSN 1549-3644

(C) 2010 Science Publications

\title{
Interpolating Operators for Multiapproximation
}

\author{
Eman Samir Bhaya \\ Department of Mathematics, College of Education, Babylon University, Iraq
}

\begin{abstract}
Problem statement: There are no simple definitions of operators for best multiapproximation and best one sided multiapproximation which work for any measurable function in $\mathrm{L}_{\mathrm{p}}$ for, $\mathrm{p}>0$. This study investigated operators that are good for best multiapproximation and best one sided multiapproximation. Approach: We first introduced some direct results related to the approximation problem of continuous functions by Hermit-Fejer interpolation based on the zeros of Chebyshev polynomials of the first or second kind in terms of the usual modulus of continuity. They were then improved to spaces $\mathrm{L}_{\mathrm{p}}$ for $\mathrm{p}<1$, in terms of the first order averaged modulus of smoothness. However, because this suffers from convergence problems, we improved and generalized these direct estimations by defining an interpolating multivariate operator $\mathrm{H}_{\mathrm{n}}(\mathrm{f})$ of measurable functions, that operator based on the zeros of Chepyshev polynomials of the first kind and prove that for any measurable function defined on $L_{p}[-1,1]^{d}$ the sequence $H_{n}(f)$ converges uniformly to $f$. Results: The resulting operators were defined for functions f such that $\mathrm{f}^{(\mathrm{k})}, \mathrm{k}=0,1, \ldots$ is of bounded variation. Then, the order of best onesided trigonometric approximation to bounded measurable functions in terms of the average modulus of smoothness was characterized. Estimates characterizing the order of best onesided approximation in terms of the k-th averaged modulus of smoothness for any function in spaces $\mathrm{L}_{\mathrm{p}}, \mathrm{p}<\infty$ were obtained. In our research we also approximated one sidedly these measurable functions in $\mathrm{L}_{\mathrm{p}}[-$ $1,1]^{\mathrm{d}}$ by defining a new operator for onesided approximation and prove a direct theorem for best one sided multiapproximation in terms of the first order averaged moduli of smoothness. Conclusion: The proposed method successfully construct operators for best multi approximation and best one sided multiapproximation for any measurable function in $\mathrm{L}_{\mathrm{p}}$ for, $\mathrm{p}>0$.
\end{abstract}

Key words: Interpolating operator, best approximation, onesided approximation, multiapproximation

\section{INTRODUCTION}

In Jishan and Ziyu (1989), introduced some results related with the approximation problem of continuous functions by Hermit-Fejer interpolation based on the zeros of Chebyshev polynomials of the first or second kind. And they proved:

If $\mathrm{f} \in \mathrm{C}[-1,1]$, then:

$$
\begin{aligned}
& \left|\mathrm{H}_{\mathrm{n}}^{1}(\mathrm{f}, \mathrm{x})-\mathrm{f}(\mathrm{x})\right| \leq \mathrm{c}\left(\frac{1}{\mathrm{n}}\left|\mathrm{T}_{\mathrm{n}}(\mathrm{x}) \mathrm{T}_{\mathrm{n}-1}(\mathrm{x})\right|\left|\mathrm{f}^{\prime}(\mathrm{x})\right|\right. \\
& +\frac{1}{\mathrm{n}}\left|\mathrm{T}_{\mathrm{n}}(\mathrm{x})\right| \omega\left(\mathrm{f}^{\prime}, \frac{\left|\mathrm{T}_{\mathrm{n}}(\mathrm{x})\right|}{\mathrm{n}}\right) \\
& +\frac{1}{\mathrm{n}}\left|\mathrm{T}_{\mathrm{n}}(\mathrm{x})\right|^{2} \sum_{\mathrm{k}=1}^{\mathrm{n}}\left(\frac{\sqrt{1+\mathrm{x}^{2}}}{\mathrm{k}}+\frac{1}{\mathrm{k}^{2}}\right) \omega\left(\mathrm{f}^{\prime}, \frac{\sqrt{1+\mathrm{x}^{2}}}{\mathrm{k}}+\frac{1}{\mathrm{k}^{2}}\right)
\end{aligned}
$$

where, $\omega\left(f^{\prime}, \frac{\sqrt{1+x^{2}}}{k}+\frac{1}{k^{2}}\right)$ is the usual modulus of smoothness for f'.
Jassim and Tahir (1996) introduced a research to improve the above estimation to the spaces $L_{p}[-1,1]$ for $\mathrm{p} \geq 1$, they proved:

$$
\left\|H_{n}^{1}(f, x)-f(x)\right\|_{P} \leq \frac{c}{n} \sum_{k=1}^{n} \tau\left(f^{\prime} \frac{k \sqrt{1+x^{2}}}{n}+\left(\frac{k}{n}\right)^{2}\right)_{p}
$$

where, $\tau\left(\mathrm{f}, \frac{\mathrm{k} \sqrt{1+\mathrm{x}^{2}}}{\mathrm{n}}+\left(\frac{\mathrm{k}}{\mathrm{n}}\right)^{2}\right)_{\mathrm{p}}$ is the averaged modulus of smoothness for $\mathrm{f}$ of the first order.

But these convergence are all not uniformly and the right hand side may not converge to zero. For this reason we shall introduce an improvement and a generalization for the estimations above. That by defining an interpolating multivariate operator $\mathrm{H}_{\mathrm{n}}(\mathrm{f})$ of the measurable functions, that operator based on the zeros of Chepyshev polynomials of the first kind and prove that for any measurable function defined on $\mathrm{L}_{\mathrm{p}}[-$ $1,1]^{d}$ the sequence $H_{n}(f)$ converges uniformly to $f$. 


\section{MATERIALS AND METHODS}

Theorem 1: For any measurable function $\mathrm{f}$ in $\mathrm{L}_{\mathrm{p}}[-1,1]^{\mathrm{d}}$ $0<\mathrm{p}<\infty$ we have:

$$
\|\mathrm{f}-\mathrm{H}(\mathrm{f})\|_{\mathrm{p}} \leq \mathrm{c}(\mathrm{p}, \mathrm{d}) \tau\left(\mathrm{f}, \frac{1}{\mathrm{n}}\right)_{\mathrm{p}}
$$

where, $c(p, d)$ is a constant depending on $p$ and d only.

The first authors on onesided approximation, Freud (1955) and Ganelius (1956), used operators for constructing polynomials of one sided approximation. These operators were defined for functions $f$ such that $\mathrm{f}^{(\mathrm{k})}, \mathrm{k}=0,1, \ldots$ is of bounded variation. Also Andreev et al. (1979); Popov (1977) and Popov and Andrev (1978) characterized the order of best onesided trigonometric approximation to bounded measurable functions in terms of the average moduli. After that Stojanova (1988); Popov and Andrev (1978) and Hristov and Ivanov (1988; 1990a; 1990b) obtained estimates characterizing the order of best onesided approximation in terms of the kth averaged modulus of smoothness for any function in the spaces $L_{p}, p<\infty$. In this study we also approximate one sidedly these measurable functions in $\operatorname{Lp}[-1,1]^{\mathrm{d}}$ by defining a new operator for onesided approximation and prove the following result:

Theorem 2: For any bounded and measurable functions in $\mathrm{L}_{\mathrm{p}}[-1,1]^{\mathrm{d}} 0<\mathrm{p}<\infty$ :

$$
\left\|H^{+}(f)-H^{-}(f)\right\|_{p} \leq c(p, d) \tau\left(f, \frac{1}{n}\right)_{p}
$$

Let $T_{n}(x)=\cos \left(n \cos ^{-1}(x)\right), x \in[-1,1]$ be the first kind Chebyshev polynomial and let: $\mathrm{x}_{\mathrm{k}}=\cos \theta_{\mathrm{k}}, \theta_{\mathrm{k}}=\frac{(2 \mathrm{k}-1) \pi}{2 \mathrm{n}}, \mathrm{k}=1,2, \ldots \ldots, \mathrm{n}$ be its zeros. Now for a function $\mathrm{f} \in \mathrm{C}[-1,1]^{\mathrm{d}}$, we denote by $\mathrm{H}_{2 \mathrm{n}-1}^{\mathrm{d}}(\mathrm{f}, \mathrm{x})$ the interpolating polynomial based on $\left\{\mathrm{x}_{\mathrm{k}}\right\}_{\mathrm{k} \in N_{\mathrm{n}}^{d}}$ defined by:

$$
\mathrm{H}_{2 \mathrm{n}-1}^{\mathrm{d}}(\mathrm{f}, \mathrm{x})=\mathrm{H}(\mathrm{f}, \mathrm{x})=\sum_{\mathrm{k} \in \mathbb{N}_{\mathrm{n}}^{d}} \mathrm{f}\left(\mathrm{x}_{\mathrm{k}}\right) \mathrm{h}_{\mathrm{k}}(\mathrm{x})
$$

where, $\quad \mathrm{x} \in[-1,1], \quad\left(\mathrm{x}_{\mathrm{k}}, \ldots, \mathrm{x}_{\mathrm{kd}}\right)=\left(\cos \theta_{\mathrm{k}_{1}}, \theta_{\mathrm{k}_{\mathrm{d}}}\right)$, $\theta_{\mathrm{k}_{\mathrm{s}}} \frac{\left(2 \mathrm{k}_{\mathrm{s}}-1\right) \pi}{2 \mathrm{n}} \mathrm{x}\left(\mathrm{x}_{1}, \ldots, \mathrm{x}_{\mathrm{d}}\right)=\left(\cos \theta_{1}, \ldots, \cos \theta_{\mathrm{d}}\right)=\Phi(\theta)$, $\theta\left(\theta_{1}, \ldots, \theta_{\mathrm{d}}\right), \theta_{\mathrm{s}} \in[0, \pi], \mathrm{k}=\left(\mathrm{k}_{1}, \ldots \mathrm{k}_{\mathrm{d}}\right), \Omega=[0, \pi]^{\mathrm{d}}, \mathrm{h}_{\mathrm{k}}(\mathrm{x})=$ $\mathrm{I}_{\mathrm{k}_{1}}\left(\mathrm{x}_{1}\right), \ldots, \mathrm{I}_{\mathrm{k}_{\mathrm{d}}}\left(\mathrm{x}_{\mathrm{d}}\right)$
For $\mathrm{x} \in \mathrm{R}^{\mathrm{d}}$, let us define $|\mathrm{x}|=\max \left\{\left|\mathrm{x}_{\mathrm{i}}\right|, \mathrm{i}=1, \ldots, \mathrm{d}\right\}$, $I_{k_{s}}\left(x_{s}\right)=\left(1-x_{s} x_{k_{s}}\right)^{\ell+1}\left(\frac{T_{n}\left(x_{s}\right.}{n\left(x_{s}-x_{k_{s}}\right)}\right)^{2 \ell+2}, S=1, \ldots, d$ and $\ell$ $\in$ Z. It is clear that $\mathrm{H}(\mathrm{f}$, .) $)$ is a polynomial of degree $(2 \mathrm{n}-1)(\ell+1)$ d. $\mathrm{T}_{\mathrm{n}}\left(\mathrm{x}_{\mathrm{s}}\right)=\cos \left(\mathrm{n} \cos ^{-1}\left(\mathrm{x}_{\mathrm{s}}\right)\right)$. We can easily prove that for each $\mathrm{k}_{\mathrm{s}}, \mathrm{I}_{\mathrm{k}_{\mathrm{s}}}\left(\mathrm{x}_{\mathrm{k}_{\mathrm{s}}}\right)$ and therefore:

$\mathrm{H}\left(\mathrm{f}, \mathrm{x}_{\mathrm{k}}\right)=\left(\mathrm{f}\left(\mathrm{x}_{\mathrm{k}}\right)\right.$

and

$\mathrm{D}^{\alpha} \mathrm{H}\left(\mathrm{f}, \mathrm{x}_{\mathrm{k}}\right)=0$

Where:

$\mathrm{D}^{\alpha}=\mathrm{D}_{1}, \mathrm{D}_{2}, \ldots \mathrm{D}_{\mathrm{n}}$

$\alpha=\left(\alpha_{1}, \ldots, \alpha_{d}\right), \alpha_{1}=\ldots=\alpha_{d}=1$

$\mathrm{D}_{1}=\frac{\partial^{\alpha_{1}} \mathrm{H}}{\partial \mathrm{x}_{\alpha_{1}}}$

$\mathrm{D}_{2}=\frac{\partial^{\alpha_{2}} \mathrm{H}}{\partial \mathrm{x}_{\alpha_{2}}}, \ldots ., \mathrm{D}_{\mathrm{d}}=\frac{\partial^{\alpha_{\mathrm{d}}} \mathrm{H}}{\partial \mathrm{x}_{\alpha_{\mathrm{d}}}}$

In the light of (1) and (2) we have this polynomial is uniquely characterized, so that $\mathrm{H}(1, \mathrm{k}) \sum_{\mathrm{k} \in \mathrm{N}_{\mathrm{n}}^{\mathrm{d}}} \mathrm{h}_{\mathrm{k}}(\mathrm{x})=1$.

Let $\Delta_{h}^{k} f(x)$ denote the kth finite difference with step $h$ of $f$ in the point $x$. We denote by:

$$
\omega_{\mathrm{k}}(\mathrm{f}, \mathrm{x}, \delta)=\sup \left\{\left|\Delta_{\mathrm{h}}^{\mathrm{k}} \mathrm{f}(\mathrm{y})\right|: \mathrm{y}, \mathrm{y}+\mathrm{kh} \in \mathrm{N}\left(\frac{\mathrm{k} \delta}{2}, \mathrm{x}\right)\right\}
$$

where, $N\left(\frac{\mathrm{k} \delta}{2}, \mathrm{x}\right)=\left\{\mathrm{y} \in \mathrm{R}^{\mathrm{d}}:\|\mathrm{y}-\mathrm{x}\|<\frac{\mathrm{k} \delta}{2}\right\}, \mathrm{d}, \mathrm{k} \in \in \mathrm{N}, \delta>0$, the local modulus of $f$. Two global moduli of the function $\mathrm{f}$ will be used, the usual modulus of smoothness:

$$
\omega_{\mathrm{k}}(\mathrm{f}, \delta)_{\mathrm{L}_{\left.\mathrm{p}(1-1,1]^{d}\right)}}=\sup \left\{\left\|\Delta_{\mathrm{h}}^{\mathrm{k}} \mathrm{f}(.)\right\|_{\mathrm{L}_{\mathrm{p}\left(\left(-1,1, \mathrm{~d}^{\mathrm{d}}\right)\right.}}:|\mathrm{h}| \leq \delta\right\}
$$

and the average modulus of smoothness:

$$
\tau_{\mathrm{k}}(\mathrm{f}, \delta)_{\mathrm{L}_{\mathrm{p}\left(-1,1, I^{\mathrm{d}}\right)}}=\| \omega_{\mathrm{k}}\left(\mathrm{f}, \ldots, \delta \|_{\mathrm{L}_{\left.\mathrm{p}(1-1,1)^{d}\right)}}\right.
$$

The properties of $\omega_{\mathrm{k}}$ are assumed to be known. Some properties of $\tau_{\mathrm{k}}$ are given in (Sendov and Popov, 1991). 
In the sequel we denote by $\mathrm{c}$ an absolute constant which may vary from one occurrence to another even in the same line. Similarly c(.) will denote a constant which depends on a specific parameter but may change from one occurrence to another.

\section{RESULTS AND DISCUSSION}

The first aim of this part is to prove the following direct theorem for best multi approximation:

Theorem 3: For any measurable function $f$ in $L_{p}[-$ $1,1]^{\mathrm{d}}, \infty<\mathrm{p} \leq 1$, there exists a polynomial $\mathrm{H}(\mathrm{f})$ such that:

$$
\|f-H(f)\|_{p} \leq c(p, d) \tau\left(f, \frac{1}{n}\right)_{L_{p\left(1-1,11^{d}\right)}}
$$

To prove our third theorem we need the following assertions:

Lemma 1: Let $0<\lambda t \leq \operatorname{diam} \Omega$, then Hristov and Ivanov (1988):

$$
\tau_{\mathrm{k}}(\mathrm{f}, \lambda \mathrm{t}) \mathrm{L}_{\mathrm{p}(\Omega)} \leq \mathrm{c} \lambda^{\ell} \tau_{\mathrm{k}}(\mathrm{f}, \mathrm{t}) \mathrm{L}_{\mathrm{p}(\Omega)}
$$

where, $\ell=\max \{\mathrm{k}, \mathrm{m}\}$ and $\mathrm{m}=\left[\frac{\mathrm{d}}{\mathrm{p}}\right]+1([$.$] -integer part )$.

Lemma 2: See Hristov and Ivanov (1988):

$$
\int_{-\pi}^{\pi}\left(\frac{\sin \frac{\mathrm{nv}}{2}}{\sin \frac{\mathrm{v}}{2}}\right)^{2 \ell+2}|\mathrm{v}|^{\mathrm{r}} \mathrm{dv} \leq \mathrm{cn}^{-\mathrm{r}}, \quad \mathrm{r}=0,1, \ldots .2 \ell
$$

\section{Lemma 3:}

$$
\int_{0}^{\pi} \frac{1}{\mathrm{n}^{\ell \ell+1}}\left(\frac{\sin \frac{\mathrm{nv}}{2}}{\sin \frac{\mathrm{v}}{2}}\right) \mathrm{dv} \leq \frac{\mathrm{c}(\zeta)}{\mathrm{n}^{2 \ell+1}}, \quad \xi \mid \in[0,1]
$$

Proof: The lemma is easily proved by the properties of the trigonometric functions and the Bonnets (Gupta and Rani 1989) mean value theorem.

Lemma 4: Sendov and Popov (1991). For any bounded and measurable function $f$ on $[a, b]$ we have:

$$
\int_{a}^{b} f(x) d x \approx \frac{b-a}{n} \sum_{i=1}^{n} f\left(x_{i}\right), \quad x_{i}=a+(b-a)(2 i-1) 2 n
$$

\section{The proof of Theorem 3:}

$$
\left.|f(x)-H(f, x)|=\mid \sum_{k \in N_{n}^{d}} f(x)-f\left(x_{k}\right)\right) H_{k}(x) \mid
$$

Let $\operatorname{cox} \theta_{\mathrm{s}}$ and $\left(\cos \theta_{1}, \ldots, \cos \theta_{\mathrm{d}}\right)=\Phi(\theta), \mathrm{x} \in[-1,1]^{\mathrm{d}}$ and $\theta \in[\theta, \pi]^{\mathrm{d}}$. Then:

$$
\begin{aligned}
& |f(x)-H(f, x)| \\
& \leq \sum_{k \in N_{n}^{d}}\left|f(x)-f\left(x_{k}\right)\right| \prod_{s=1}^{d}\left|1-x_{s} x_{k_{s}}\right|^{\ell+1}\left(\frac{\cos n \theta_{s}}{n\left(\cos \theta_{s}-\cos \theta_{k_{s}}\right)}\right)^{2 \ell+2}
\end{aligned}
$$

Then since $\sin \theta_{\mathrm{s}} \geq 0, \sin \theta_{\mathrm{k}_{\mathrm{s}}} \geq 0, \cos n \theta_{\mathrm{k}_{\mathrm{s}}}=0$, so:

$$
\begin{aligned}
& |f(x)-H(f, x)| \\
& \leq c \sum_{k \in N_{n}^{d}}\left|f(x)-f\left(x_{k}\right)\right| \prod_{s=1}^{d}\left|1-\cos \left(\theta_{s}-\theta_{k_{s}}\right)\right|^{\ell+1} \\
& \left(\left.\frac{\sin \left(\frac{n\left(\theta_{s}-\theta_{k_{s}}\right)}{2}\right)}{n \sin \left(\frac{\theta_{s}-\theta_{k_{s}}}{2}\right)}\right|^{2 \ell+2}\right. \\
& \leq \mathrm{c} \sum_{k \in N_{n}^{d}}\left|f(x)-f\left(x_{k}\right)\right| \prod_{s=1}^{d}\left(\frac{\sin \left(\frac{n\left(\theta_{s}-\theta_{k_{s}}\right)}{2}\right)}{n \sin \left(\frac{\theta_{s}-\theta_{k_{s}}}{2}\right)}\right)^{2 \ell+2}
\end{aligned}
$$

Let us assume that:

$$
\mathrm{J}_{\ell, \mathrm{n}}\left(\theta_{\mathrm{s}}-\theta_{\mathrm{k}_{\mathrm{s}}}\right)=\frac{\mathrm{c}}{\mathrm{n}^{2 \ell+1}}\left(\frac{\sin \left(\frac{\mathrm{n}\left(\theta_{\mathrm{s}}-\theta_{\mathrm{k}_{\mathrm{s}}}\right)}{2}\right)}{\mathrm{n} \sin \left(\frac{\theta_{\mathrm{s}}-\theta_{\mathrm{k}_{\mathrm{s}}}}{2}\right)}\right)
$$

and

$$
\mathrm{I}_{\ell, \mathrm{n}}\left(\theta-\theta_{\mathrm{k}}\right)=\mathrm{J}_{\ell, \mathrm{n}}\left(\theta_{1}-\theta_{\mathrm{k} 1}\right) \ldots . \mathrm{J}_{\ell, \mathrm{n}}\left(\theta_{\mathrm{d}}-\theta_{\mathrm{kd}}\right)
$$

Then:

$$
|\mathrm{f}(\mathrm{x})-\mathrm{H}(\mathrm{f}, \mathrm{x})| \leq \mathrm{c}\left(\frac{1}{\mathrm{n}}\right)^{\mathrm{d}}|\mathrm{f}(\Phi(\theta))-\mathrm{f}(\Phi(\mathrm{t}))| \mathrm{J}_{\ell, \mathrm{n}}\left(\theta_{\mathrm{k}}\right)
$$

Then by Lemma 4 we have: 


$$
|\mathrm{f}(\mathrm{x})-\mathrm{H}(\mathrm{f}, \mathrm{x})| \leq \mathrm{c} \int_{\Omega}|\mathrm{f}(\Phi(\theta))-\mathrm{f}(\Phi(\mathrm{t}))| \mathrm{J}_{\ell, \mathrm{n}}(\mathrm{t}) \mathrm{dt}
$$

Thus:

$$
\begin{aligned}
& \|\mathrm{f}(\mathrm{x})-\mathrm{H}(\mathrm{f}, \mathrm{x})\|_{\mathrm{p}} \leq \mathrm{c}\left(\int_{\Omega}\left(\int_{\Omega} \mid \mathrm{f}(\Phi(\theta))-\mathrm{f}\left(\Phi(\mathrm{t}) \mid \mathrm{J}_{\ell, \mathrm{n}}(\mathrm{t}) \mathrm{dt}\right)^{\mathrm{p}} \mathrm{d} \theta\right)^{\frac{1}{\mathrm{p}}}\right. \\
& \leq \mathrm{c}\left(\int_{\Omega}\left(\int_{\Omega} \omega(\mathrm{f}(\Phi(\theta)), \theta,|\theta-\mathrm{t}|)\right)^{\mathrm{p}} \mathrm{d} \theta\right)^{\frac{1}{\mathrm{p}}} \mathrm{J}_{\ell, \mathrm{n}}(\mathrm{t}) \mathrm{dt} \\
& =\mathrm{c} \int_{\Omega} \tau(\mathrm{f}(\Phi(\theta)), \mid \theta-\mathrm{t}) \mid \mathrm{L}_{\mathrm{p}(\Omega)} \mathrm{J}_{\ell, \mathrm{n}}(\mathrm{t}) \mathrm{dt}
\end{aligned}
$$

Lemma 1 and Lemma 2, lead to:

$$
\begin{aligned}
& \|f(x)-H(f, x)\|_{p} \leq \mathrm{c}(\zeta) \tau\left(f(\Phi(\theta)), \frac{1}{n}\right)_{L_{p(\Omega)}} \\
& =c(\zeta) \tau\left(f(x), \frac{1}{n}\right)_{L_{p}\left([-1,1]^{d}\right)}
\end{aligned}
$$

For the case $0<p<1$, we can prove theorem 1 by follows the same lines above and using the inequality (Carathers, 2005):

$$
\|\mathrm{f}+\mathrm{g}\|_{\mathrm{p}}^{\mathrm{p}} \leq\|\mathrm{f}\|_{\mathrm{p}}^{\mathrm{p}}+\|\mathrm{g}\|_{\mathrm{p}}^{\mathrm{p}}
$$

In the second result of present study we construct a one-sided operator using $\mathrm{H}(\mathrm{f}, \mathrm{x})$ as follows:

$$
\mathrm{H}^{+,-}(\mathrm{f}, \mathrm{x})=\mathrm{H}(\mathrm{f}, \mathrm{x}) \pm \sum_{\mathrm{k} \in \mathrm{N}_{\mathrm{n}}^{\mathrm{d}}} \psi(\mathrm{x}) \sup _{\left|\mathrm{r}(\mathrm{y})-\frac{\pi(2 j-1)}{2 \mathrm{n}}\right| \frac{\pi}{2 \mathrm{n}}}|\mathrm{f}(\mathrm{y})-\mathrm{H}(\mathrm{f}, \mathrm{y})|
$$

Where:

$\mathrm{r}(\mathrm{y})=\left(\cos ^{-1} \mathrm{y}_{1}, \ldots, \cos ^{-1} \mathrm{y}_{\mathrm{d}}\right)$

$\mathrm{y}_{1}=\cos \theta_{1}, \ldots, \mathrm{y}_{\mathrm{d}}=\cos \theta_{\mathrm{d}}$

$$
\psi\left(x_{s}\right)=\prod_{i=1}^{d}\left(\frac{T_{n}\left(x_{s}\right)}{n\left(x_{s}-x_{s i}\right)}\right)^{2}\left(1-\omega_{i} z_{i}\right)^{\ell}, T_{n}\left(x_{s}\right)=\cos \left(n \cos ^{-1} x_{s}\right)
$$$$
\omega_{\mathrm{i}}=\cos \frac{\pi}{\mathrm{n}}, \mathrm{z}_{\mathrm{i}}=\cos \frac{\pi}{2 \mathrm{n}}, \mathrm{x}_{\mathrm{s}} \in[-1,1]=\eta,
$$$$
\mathrm{x}_{\mathrm{si}}=\cos \theta_{\mathrm{si}}, \theta_{\mathrm{si}}=\frac{\left(2 \mathrm{~s}_{\mathrm{i}}-1\right) \pi}{2 \mathrm{n}}
$$

and

$$
|\mathrm{x}|=\sup _{1 \leq \mathrm{i} \leq \mathrm{d}}\left|\mathrm{x}_{\mathrm{i}}\right|
$$

This operator is a one-sided operator, since:

- The polynomials $H^{+-}(f, x)$ of degree $(2 n-1)(1+1) d$

- $\mathrm{H}^{-}(\mathrm{f}, \mathrm{x}) \leq \mathrm{f}(\mathrm{x}) \leq \mathrm{H}^{+}(\mathrm{f}, \mathrm{x}), \mathrm{x} \in[-1,1]^{\mathrm{d}}=\eta$. Because of the positivity of $\psi\left(x_{s}\right)$, for each $s=1,2, \ldots, d$

Then we introduce our second result.

Theorem 4: For any bounded function $\mathrm{f}$ in $\mathrm{L}_{\mathrm{p}}[-1,1]=$ $\eta, \infty<p \leq 1$, there exist two polynomials $\mathrm{H}^{+}(\mathrm{f})$ and $\mathrm{H}^{-}(\mathrm{f})$ such that:

$$
\left\|H^{+}(f)-H^{-}(f)\right\|_{p} \leq c(p, d) \tau\left(f, \frac{1}{n}\right)_{L_{p(n)}}
$$

For the proof of this theorem we need the following Lemma:

\section{Lemma 5:}

$$
\begin{aligned}
& \psi\left(\mathrm{x}_{\mathrm{s}}\right)=\prod_{\mathrm{i}=1}^{\mathrm{d}}\left(\frac{\mathrm{T}_{\mathrm{n}}\left(\mathrm{x}_{\mathrm{s}}\right)}{\mathrm{n}\left(\mathrm{x}_{\mathrm{s}}-\mathrm{x}_{\mathrm{si}}\right)}\right)^{2}\left(\mathrm{I}-\omega_{\mathrm{i}} \mathrm{z}_{\mathrm{i}}\right)^{\ell} \leq \\
& \mathrm{c} \prod_{\mathrm{i}=1}^{\mathrm{d}}\left(\frac{\sin \left(\frac{\mathrm{n}\left(\theta_{\mathrm{s}}-\theta_{\mathrm{s}_{\mathrm{i}}}\right)}{2}\right)}{\mathrm{n} \sin \left(\frac{\left(\theta_{\mathrm{s}}-\theta_{\mathrm{s}_{\mathrm{i}}}\right)}{2}\right)}\right) \sin \left(\frac{\pi}{2 \mathrm{n}}\right)^{2 \ell} \leq\left(\frac{\pi}{2 \mathrm{n}}\right)^{2 \ell}
\end{aligned}
$$

Proof: Let $x_{\mathrm{si}}=\cos \theta_{\mathrm{s}_{\mathrm{i}}}, \mathrm{x}_{\mathrm{s}}=\cos \theta_{\mathrm{s}} \theta_{\mathrm{s}_{\mathrm{i}}}=\frac{\left(2_{\mathrm{s}_{\mathrm{i}}}-1\right) \pi}{2 \mathrm{n}}$, then $\psi\left(x_{\mathrm{s}}\right)=\prod_{\mathrm{i}=1}^{\mathrm{d}}\left(\frac{\cos \theta_{\mathrm{s}}}{\mathrm{n}\left(\cos \theta_{\mathrm{s}}-\cos \theta_{\mathrm{s}_{\mathrm{i}}}\right)}\right)^{2}\left(1-\cos \frac{\pi}{2} \cos \frac{\pi}{2 \mathrm{n}}\right)^{\ell} \cos \theta_{\mathrm{si}}=$ 0 implies:

$$
\begin{aligned}
& \psi\left(\mathrm{x}_{\mathrm{s}}\right)=\prod_{\mathrm{i}=1}^{\mathrm{d}}\left(\frac{\cos \theta_{\mathrm{s}}-\cos n \theta_{\mathrm{si}}}{\mathrm{n}\left(\cos \theta_{\mathrm{s}}-\cos \theta_{\mathrm{si}}\right)}\right)^{2}\left(1-\cos \frac{\pi}{2} \cos \frac{\pi}{2 \mathrm{n}}\right)^{\ell} \\
& \leq \prod_{\mathrm{i}-1}^{\mathrm{d}}\left(\frac{\sin \left(\frac{\mathrm{n}\left(\theta_{\mathrm{s}}-\theta_{\mathrm{si}}\right)}{2}\right)}{\mathrm{n} \sin \left(\frac{\left(\theta_{\mathrm{s}}-\theta_{\mathrm{si}}\right)}{2}\right)}\right)^{2}\left(1-\cos \frac{\pi}{2} \cos \frac{\pi}{2 \mathrm{n}}+\sin \frac{\pi}{\mathrm{n}} \sin \frac{\pi}{2 \mathrm{n}}\right)^{\ell} \\
& <\prod_{\mathrm{i}-1}^{\mathrm{d}}\left(\frac{\sin \left(\frac{\left(\theta_{\mathrm{s}}-\theta_{\mathrm{si}}\right)}{2}\right)}{\mathrm{n} \sin \left(\frac{\left(\theta_{\mathrm{s}}-\theta_{\mathrm{si}}\right)}{2}\right)}\right)^{2}\left(1-\cos \left(\frac{\pi}{2}+\frac{\pi}{2 \mathrm{n}}\right)\right)^{\ell} \\
& =\prod_{\mathrm{i}-1}^{\mathrm{d}}\left(\frac{\sin \left(\frac{\left(\theta_{\mathrm{s}}-\theta_{\mathrm{si}}\right)}{2}\right)}{\mathrm{n} \sin \left(\frac{\left(\theta_{\mathrm{s}}-\theta_{\mathrm{si}}\right)}{2}\right)}\right)^{2} \sin ^{2 \ell} \frac{\pi}{2 \mathrm{n}} \leq 2^{\mathrm{d}} \sin ^{2 \ell \mathrm{d}} \frac{\pi}{2 \mathrm{n}}
\end{aligned}
$$


which completes the proof.

\section{Proof of Theorem 4:}

$$
\begin{aligned}
& \sup _{\left|\mathrm{r}(\mathrm{y})-\frac{\pi(2 \mathrm{j}-1)}{2 \mathrm{n}}\right| \leq \frac{\pi}{2 \mathrm{n}} \mid} \mid \mathrm{f}(\mathrm{y})-\mathrm{H}(\mathrm{f}, \mathrm{y}) \leq\left(\frac{\mathrm{c}}{\mathrm{n}}\right)^{\mathrm{d}} \sup _{\left|\mathrm{r}(\mathrm{y})-\frac{\pi(2 \mathrm{j}-1)}{2 \mathrm{n}}\right| \leq \frac{\pi}{2 \mathrm{n}}} \\
& \sum_{\mathrm{k} \in \mathbb{N}_{\mathrm{n}}^{\mathrm{d}}}\left|\mathrm{f}(\mathrm{y})-\mathrm{f}\left(\mathrm{x}_{\mathrm{k}}\right)\right| \mathrm{J}_{\ell, \mathrm{n}}\left(\frac{(2 \mathrm{k}-1) \pi}{2 \mathrm{n}}\right)
\end{aligned}
$$

Now let $\mathrm{x}_{\mathrm{ks}},=\cos \theta_{\mathrm{k}}, \theta_{\mathrm{k}}=\frac{\left(2 \mathrm{k}_{\mathrm{s}}-1\right) \pi}{2 \mathrm{n}}, \quad \mathrm{r}(\mathrm{y})=$ $\left(\cos ^{-1} y_{1}, \ldots, \cos ^{-1} y_{d}\right), y_{1}=\cos \theta^{\star}{ }_{1}, \ldots, y_{d}=\cos \theta^{\circ}$. Then:

$$
\begin{aligned}
& \sup _{\mid \mathrm{r}(\mathrm{y}) \frac{(2 \mathrm{j}-1)}{2 \mathrm{n}} \leq \frac{\pi}{2 \mathrm{n}}}|\mathrm{f}(\mathrm{y})-\mathrm{H}(\mathrm{f}, \mathrm{y})| \leq\left(\frac{\mathrm{c}}{\mathrm{n}}\right)^{\mathrm{d}} \sup _{\left|\mathrm{r}(\mathrm{y}) \frac{\pi(2 \mathrm{j}-1)}{2 \mathrm{n}}\right| \leq \frac{\pi}{2 \mathrm{n}}} \\
& \int_{\Omega=[0, \pi]^{\mathrm{d}}}\left|\mathrm{f}\left(\Phi\left(\theta^{\prime}\right)\right)-\mathrm{f}(\Phi(\mathrm{t}))\right| \mathrm{J}_{\ell, \mathrm{n}}(\mathrm{t}) \mathrm{dt} \\
& \leq\left(\frac{\mathrm{c}}{\mathrm{n}}\right)^{\mathrm{d}} \sup _{\left|\mathrm{r}(\mathrm{y}) \frac{\pi(2 \mathrm{j}-1)}{2 \mathrm{n}}\right| \leq \frac{\pi}{2 \mathrm{n}} \Omega} \int \omega\left(\mathrm{f}^{\circ} \Phi, \frac{\pi(2 \mathrm{j}-1)}{2 \mathrm{n}}, \frac{\pi}{2 \mathrm{n}}\right) \mathrm{J}_{\ell, \mathrm{n}}(\mathrm{t}) \mathrm{dt} \\
& \leq\left(\frac{\mathrm{c}}{\mathrm{n}}\right)^{\mathrm{d}} \sup _{\left|\mathrm{r}(\mathrm{y}) \frac{\pi(2 j-1)}{2 \mathrm{n}}\right| \leq \frac{\pi}{2 \mathrm{n}} \Omega} \int \omega\left(\mathrm{f}^{\circ} \Phi, \frac{\pi(2 \mathrm{j}-1)}{2 \mathrm{n}}, \frac{\pi}{2 \mathrm{n}}+|\mathrm{t}|\right) \mathrm{J}_{\ell, \mathrm{n}}(\mathrm{t}) \mathrm{dt}
\end{aligned}
$$

Then:

$$
\begin{aligned}
& \left\|\mathrm{H}^{+}(\mathrm{f})-\mathrm{H}^{-}(\mathrm{f})\right\|_{\mathrm{p}}=2\left\|\sum_{j \in \mathbb{N}_{\mathrm{n}}^{d}} \psi(\mathrm{x}) \sup _{\mid \mathrm{r}(\mathrm{y}) \frac{\pi(2 \mathrm{j}-1)}{2 \mathrm{n}} \leq \leq \frac{\pi}{2 \mathrm{n}}} \mid \mathrm{f}(\mathrm{y})-\mathrm{H}(\mathrm{f}, \mathrm{y})\right\| \|_{\mathrm{p}} \\
& \leq 2\left\|\sum_{j \in \mathrm{N}_{\mathrm{d}}^{d}} \psi(\mathrm{x}) \int_{\Omega} \psi(\mathrm{x}) \omega\left(\mathrm{f}^{\circ} \Phi, \frac{\pi(2 \mathrm{j}-1)}{2 \mathrm{n}}, \frac{\pi}{2 \mathrm{n}}+|\mathrm{t}|\right) \mathrm{J}_{\ell, \mathrm{n}}(\mathrm{t}) \mathrm{dt}\right\|_{\mathrm{p}}
\end{aligned}
$$

Then realize on Lemma 5, to obtain:

$$
\begin{aligned}
& \left\|H^{+}(f)-H^{-}(f)\right\|_{p}=2\left\|\int_{\int_{2} \in N_{n}^{d}}\left(\begin{array}{l}
\left.\frac{\pi}{2 n}\right) \omega \\
\frac{\pi}{2 n}+|t|
\end{array}\right) J_{\ell, n}(t) d t\right\|_{p} \\
& \leq \mathrm{c}\left\|\int_{\Omega \Omega} \int_{\Omega}\left(\frac{\pi}{2 \mathrm{n}}\right)^{\mathrm{d} \ell} \omega\left(\mathrm{f}^{\circ} \Phi, \theta, \frac{\pi}{2 \mathrm{n}}+|\mathrm{t}|\right) \mathrm{d} \theta \mathrm{J}_{\ell, \mathrm{n}}(\mathrm{t}) \mathrm{d}\right\|_{\mathrm{p}} \\
& \leq \mathrm{c}(\mathrm{p}, \mathrm{d})\left(\frac{1}{\mathrm{n}}\right)^{\mathrm{d} \ell}\left\|\iint_{\Omega}\right\| \omega\left(\mathrm{f}^{\circ} \Phi, \theta, \frac{\pi}{2 \mathrm{n}}+|\mathrm{t}|\right)\left\|_{\mathrm{L}_{\mathrm{p}(\Omega)}} \mathrm{J}_{\ell, \mathrm{n}}(\mathrm{t}) \mathrm{dt}\right\| \|_{\mathrm{P}} \\
& \leq \mathrm{c}(\mathrm{p}, \mathrm{d})\left(\frac{1}{\mathrm{n}}\right)^{\mathrm{d} \ell}\left\|\int_{\Omega} \tau\left(\mathrm{f}^{\circ} \Phi, \theta, \frac{\pi}{2 \mathrm{n}}+|\mathrm{t}|\right)_{\mathrm{L}_{\mathrm{p}(\Omega)}} \mathrm{J}_{\ell, \mathrm{n}}(\mathrm{t}) \mathrm{dt}\right\|_{\mathrm{p}}
\end{aligned}
$$

By virtue of Lemma 1 and Lemma 2, we immediately have:

$$
\begin{aligned}
& \left.\left\|H^{+}(f)-H^{-}(f)\right\|_{p} \leq \mathrm{c}(\mathrm{p}, \mathrm{d})\left(\int_{\Omega} \tau\left(\mathrm{f}_{\Omega}^{\circ} \tau, \frac{\pi}{2 \mathrm{n}}\right)_{\mathrm{L}_{\mathrm{p}(\Omega)}} \mathrm{J}_{\ell, \mathrm{n}}(\mathrm{t}) \mathrm{dt}+\mathrm{f}^{\circ} \Phi, \frac{|\mathrm{t}| \mathrm{n}}{\mathrm{n}}\right)_{\mathrm{L}_{\mathrm{p}(\Omega)}} \mathrm{J}_{\ell, \mathrm{n}}(\mathrm{t}) \mathrm{dt}\right) \\
& \leq \mathrm{c}(\mathrm{p}, \mathrm{d}) \tau\left(\mathrm{f}^{\circ} \Phi, \frac{\pi}{\mathrm{n}}\right)_{\mathrm{L}_{\mathrm{p}(\Omega)}}+\int_{\Omega} \mathrm{n}^{\ell}|\mathrm{t}|^{\ell} \tau\left(\mathrm{f}^{\circ} \Phi, \frac{|\mathrm{t}| \mathrm{n}}{\mathrm{n}}\right)_{\mathrm{L}_{\mathrm{p}(\Omega)}} \\
& \mathrm{J}_{\ell, \mathrm{n}}(\mathrm{t}) \mathrm{dt} \leq \mathrm{c}(\mathrm{p}, \mathrm{d}) \tau\left(\mathrm{f}^{\circ} \Phi, \frac{1}{\mathrm{n}}\right)_{\mathrm{L}_{\mathrm{p}(\Omega)}} \leq(\mathrm{p}, \mathrm{d})\left(\mathrm{f}, \frac{1}{\mathrm{n}}\right)_{\mathrm{L}_{\mathrm{p}(n)}}
\end{aligned}
$$

\section{CONCLUSION}

In this study, we successfully constructed operators for best multiapproximation and best one sided multiapproximation for the study of any measurable function in $\mathrm{L}_{\mathrm{p}}$ for, $\mathrm{p}>0$.

\section{REFERENCES}

Andreev, A., Popov V. and B. Sendov, 1979. Jacksontype theorems for best one-sided aproximations by trigonometrical polynomials and splines. Math. Notes J., 26: 889-896. DOI: 10.1007/BF01159203

Carathers, N., 2005. A Short Course on Banach Space Theory. 3rd Edn., Bowling Green State University, USA., ISBN: 0521603722, pp: 196.

Freud, G., 1955. Uber einseitige approximation durch polynome. I. Acta Sci. Math., 16: 12-18.

Ganelius, T., 1956. On one sided approximation by trigonometrical polynomials. Math. Scand., 4: 247-258.

Gupta, S. and N. Rani, 1989. Fundamental Real Analysis. 3rd Edn., Vikas Publishing House PVT LTD., ISBN: 0-70-692819-9, pp: 306.

Hristov, V. and K. Ivanov, 1988. Operators for one sided approximation of functions. Mathematica Balkanica, 2: 374-390.

Hristov, V. and K. Ivanov, 1990a. Characterization of best approximation from below and from above. Proceeding of the Conference on Approximation Theory, (AT'90), Kecskemet, pp: 377-403.

Hristov, V. and K. Ivanov, 1990b. Realization of Kfunctionals on subsets and constrained approximation. Math. Balkanica, 4: 236-257.

Jassim, S. and M. Tahir, 1996. Approximation by Hermit-Fejer polynomial. J. Iraq Soc. Phys. Math., In Press.

Jishan, T. and W. Ziya, 1989. On the approximation of the continuous functions by some Hermit-Fejer interpolation polynomials. J. Approx. Theory Applied, 5: 63-67. DOI: 10.1007/BF02836115 
Popov, V. and A. Andrev, 1978. Steckin's type theorems for one sided trigonometrical and spline approximation. Comp. Rend. Acad. Bulg. Sci., 31: 151-154.

Popov, V., 1977. Converse theorem for the one sided trigonometrical approximations. Comp. Rend. Acad. Bulg. Sci., 30: 1529-1532.
Sendov, B. and V. Popov, 1991. The averaged moduli of smoothness: With applications in numerical methods and approximation. SIAM Rev., 33: 499-501. http://www.jstor.org/pss/2031464

Stojanova, M., 1988. The best one sided algebraic approximation in $\mathrm{L}_{\mathrm{p}}[-1,1], \quad 1 \leq \mathrm{p} \leq \infty$. Math. Balkanica, 2: 101-113. 